\title{
Trends in the Incidence of Sudden Deaths and Heart Diseases in Fukushima After the Great East Japan Earthquake
}

\author{
Mai Takiguchi, ${ }^{1} \mathrm{MD}$, Tetsuya Ohira, ${ }^{2,3} \mathrm{MD}$, Hironori Nakano, ${ }^{2,3} \mathrm{MS}$, Yui Yumiya, ${ }^{2,3} \mathrm{MPH}$, \\ Takayoshi Yamaki, ${ }^{1}$ MD, Akiomi Yoshihisa, ${ }^{1}$ MD, Kazuhiko Nakazato, ${ }^{1}$ MD, Hitoshi Suzuki, ${ }^{1}$ MD, \\ Tetsuo Ishikawa, ${ }^{2,4} \mathrm{PhD}$, Seiji Yasumura, ${ }^{2,5} \mathrm{MD}$, Kenji Kamiya ${ }^{2,6}$ MD and Yasuchika Takeishi, ${ }^{1}$ MD
}

\begin{abstract}
Summary
On March 11, 2011, a great earthquake, known as the Great East Japan Earthquake, hit northeastern Japan, resulting in a tsunami that caused a nuclear disaster, the Fukushima Daiichi Nuclear Power Plant accident, forcing about 160,000 people to evacuate. We, therefore, sought to examine the effects of this evacuation on the onset of cardiovascular diseases and sudden death (SD) in Fukushima Prefecture, three years after the earthquake. We divided the evacuation zone into two areas, whole evacuation zone (Area 1) and partial evacuation zone (Area 2), and we defined the north district of the prefecture as the control area (Area 3). We cross-referenced the death certificate data with data from the Fukushima Prefecture acute myocardial infarction registration survey. For each area, we tallied the number of people who fell into the SD, myocardial infarction (MI), and MI suspected groups. We calculated the age-adjusted incidence rates and analyzed the differences in the adjusted incidence rates across three years using a Poisson regression model. The age-adjusted death rate of the SD group was significantly higher in 2011 in all areas than in 2012 or $2013(P<0.05)$. The total death rate was higher in Area 1 in March 2011, just after the disaster, than in the other two areas. The rate of SD was also higher in Area 1 than in the other areas in March 2011. The incidence of sudden cardiac death might have increased just after the Great East Japan Earthquake in the evacuation area, but not in other areas in Fukushima Prefecture.
\end{abstract}

Key words: Disasters, Coronary artery disease, Epidemiology

(Int Heart J 2019; 60: 1253-1258)

$\mathrm{O}$ n March 11, 2011, a great earthquake, known as the Great East Japan Earthquake, hit northeastern Japan. The moment magnitude scale $(\mathrm{Mw})$ of the earthquake was 9.0, the largest earthquake that has ever been recorded in or near Japan. ${ }^{1)}$ The earthquake triggered a tsunami that caused massive damage on the Pacific coast of northeastern Japan; in addition, in Fukushima Prefecture, the earthquake and the accompanying tsunami caused the first Fukushima Daiichi Nuclear Power Plant accident. After the accident at the power plant, a large number of people living in the cities, towns, and villages around the evacuation area, as well as those living in the surrounding areas, were forced to evacuate. As a result of the earthquake, 1,614 people died and 196 people went missing, and about 160,000 people had to evacuate. ${ }^{2,3)}$

It has been previously reported that the incidence of cardiovascular diseases increases after large-scale natural disasters. For example, cardiovascular death is reported to have increased for several days after the 1981 Athens earthquake, ${ }^{4}$ and the occurrence of sudden cardiac death and unstable ventricular arrhythmias increased following the 1994 Northridge earthquake in California, USA. ${ }^{5)}$ One month after the 1995 Hanshin-Awaji earthquake, there was a sharp increase in the rate of mortality related to acute myocardial infarction (AMI) and stroke. ${ }^{6}$ In addition, in the case of the Great East Japan Earthquake, the incidences of cardiovascular disease, heart failure, pneumonia, and sudden death (SD) increased in the area of Tohoku a few months after the earthquake. ${ }^{7.8)}$ Recently, it has been reported that the occurrence of out-of-hospital cardiac arrest with a cardiac origin has increased in the first year after the earthquake.9)

The Fukushima Prefecture AMI registration survey registry study was conducted to investigate the AMI status

From the ${ }^{1}$ Department of Cardiovascular Medicine, Fukushima Medical University School of Medicine, Fukushima, Japan, ${ }^{2}$ Radiation Medical Science Center for the Fukushima Health Management Survey, Fukushima Medical University, Fukushima, Japan, ${ }^{3}$ Department of Epidemiology, Fukushima Medical University School of Medicine, Fukushima, Japan, ${ }^{4}$ Department of Radiation Physics and Chemistry, Fukushima Medical University School of Medicine, Fukushima, Japan, ${ }^{5}$ Department of Public Health, Fukushima Medical University School of Medicine, Fukushima, Japan and ${ }^{6}$ Research Institute for Radiation Biology and Medicine, Hiroshima University, Hiroshima, Japan.

This work was supported by the National Health Fund for Children and Adults Affected by the Nuclear Incident and supported by the Research Fund for Effects of Radiation on Health Status, Ministry of Environment.

Address for correspondence: Mai Takiguchi, MD, Department of Cardiovascular Medicine, Fukushima Medical University, 1 Hikarigaoka, Fukushima 9601295, Japan. E-mail: t-mai@fmu.ac.jp

Received for publication March 1, 2019. Revised and accepted June 28, 2019

Released in advance online on J-STAGE October 31, 2019.

doi: 10.1536/ihj.19-110

All rights reserved by the International Heart Journal Association. 


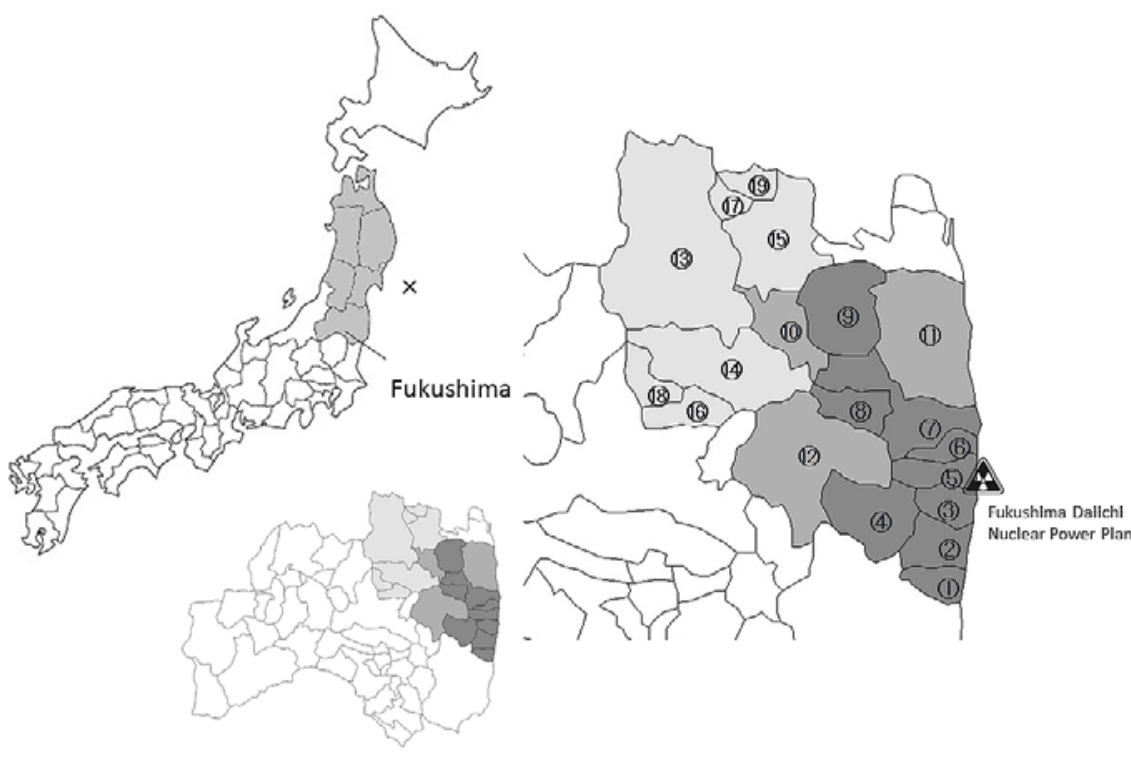

Area 1

(1) Hirono Town (2) Naraha Town (3)Tomioka Town (4) Kawauchi Town (5) Okuma Town (6) Futaba Town (7) Namie Town (8)Katsurao Villages (9)litate Villages

Area 2

(10)Kawamata Town (11)Minamisoma City (12)Tamura City Area 3 (13) Fukushima city (14) Nihonmatsu city (15)Date city (16) Motomiya-city (17)Koori city (18)Kunimi town (19)Otama Villages

Figure 1. Study area. The map on the left shows an outline of Fukushima Prefecture, Japan. X indicates the epicenter of the Great East Japan Earthquake. The map on the right shows the three study areas (19 municipalities, towns, and villages).

and improve the treatment outcomes in Fukushima Prefecture, starting from 2009. All patients admitted to hospitals in Fukushima for possible AMI treatment were enrolled in the study. The results suggested that the onset of AMI did not increase in Fukushima following the earthquake. However, it was reported that the death rate due to myocardial infarction (MI) in this prefecture was the highest in Japan. ${ }^{10)}$ The Fukushima Prefecture AMI registration survey registry applies only to those patients who were admitted to and treated at hospitals, and therefore it does not include all patients with AMI in Fukushima. In view of this, we feel that including data obtained from death certificates in the analysis would improve the accuracy of the findings. In addition, the study included data only on the location of the treating hospitals, rather than on the area in which the patients lived or were evacuated to, so it is difficult to precisely investigate the effects of proximity to the disaster. The inhabitants of Fukushima Prefecture were exposed to the stress of the earthquake, the tsunami, and the power plant accident, and the evacuation thereafter was also associated with increased cardiovascular risk factors, such as hypertension, diabetes mellitus, and dyslipidemia. ${ }^{11)}$

Although there are many studies examining the shortterm effects of the Great East Japan Earthquake, the longterm effects remain unknown. Although the evacuation zone has gradually been reduced, many people have continued living as evacuees in Fukushima Prefecture. It is, thus, important to investigate the long-term trends in the affected area. In this study, we sought to examine the effects of the evacuation on the onset of cardiovascular diseases and SD in Fukushima Prefecture, three years after the Great East Japan Earthquake.

\section{Methods}

Study population and area: The subjects of this study were Japanese men and women living in Fukushima Prefecture. Following the disaster, the Japanese government decided to declare all land within $20 \mathrm{~km}$ of the Fukushima Daiichi Nuclear Power Plant as an evacuation zone, since the total radiation exposure dose in that zone might exceed $20 \mathrm{mSv}$ per year after the accident. The government, therefore, directed all the inhabitants to evacuate from that zone.The government also created an evacuation preparation area in the zone $20-30 \mathrm{~km}$ from the power plant, in case of emergency evacuations. Finally, they prohibited entrance to the restricted area and declared it an evacuation prepared area. The evacuation zone was gradually reduced; however, for the purposes of this study, we have defined the evacuation zone as all three of these zones.

We then divided the evacuation zone into two areas, whole evacuation zone (Area 1; all residents were forced to evacuate) and partial evacuation zone (Area 2; some residents were forced to evacuate). Area 1 included the towns of Hirono, Naraha, Tomioka, Kawauchi, Okuma, Futaba, and Namie, as well as the villages of Katsurao and Iitate, whereas Area 2 included the town of Kawamata and the cities of Minamisoma and Tamura, and we defined the prefecture north district as Area 3. Area 3 included the cities of Fukushima, Nihonmatsu, Date, and Motomiya; the towns of Koori and Kunimi; and the village of Otama (not including the town of Kawamata) (Figure 1).

This study included subjects living in each area when the earthquake occurred. We examined the population in each area using the estimated population data, which were added later as a population change by the Basic Resident Register to the national census obtained in 2011 (https:// www.pref.fukushima.lg.jp/sec/11045b/15847.html). The populations in 2011, 2012, and 2013 were 78,650, 73,074, and 72,247 in Area 1; 126,474, 118,381, and 116,572 in Area 2; and 477,254, 462,793, and 460,044 in Area 3, re- 
Table I. Number of Total Deaths and SDs in Fukushima and the Target Areas from 2011 to 2013

\begin{tabular}{lrrr}
\hline & 2011 & 2012 & \multicolumn{1}{c}{2013} \\
\hline Total deaths in Fukushima & 26,177 & 23,464 & 23,547 \\
In Area 1-3 & & & \\
$\quad$ Total deaths & 1,584 & 1,409 & 1,513 \\
SDs & 994 & 797 & 849 \\
MI & 21 & 40 & 35 \\
MIS & 531 & 538 & 535 \\
\hline
\end{tabular}

SD indicates sudden death; MI, myocardial infarction; and MIS, myocardial infarction suspected.

spectively.

We then investigated the death certificates of all those who died in Fukushima Prefecture between January 2011 and December 2013, with the permission of the Ministry of Health and Welfare. The death certificates contained the following information: date of death, date of birth, sex, basic cause of death, and the area code of the deceased person. In addition, the address given for the deceased person was his/her residential address. We were, therefore, able to identify whether they were inhabitants of the evacuation zone on the basis of this address. The study protocol was approved by the ethical committee of Fukushima Medical University (no. 1586, no. 2303), and the investigation conforms with the principles outlined in the Declaration of Helsinki. The requirement for informed consent was waived.

Definition of diseases: The cause of death was coded according to the International Classification of Diseases, 10 th Revision (ICD-10). We extracted the following causes of death, as listed in the death certificates: unidentified ( $\mathrm{R}$ 96, R98, R99); heart-related causes (I30-I52), such as heart failure, arrhythmia, cardiomyopathy, valvar disease, or coronary heart disease; and MI (I20-I25). We defined deaths that occurred within 24 hours of the onset of a direct cause (excluding external causes) as "SD" cases. Death due to MI was defined as follows. Even if the disease was stated in the death certificate as MI, it was difficult to determine whether that condition was diagnosed according to the World Health Organization Multinational Monitoring of Trends and Determinants in Cardiovascular Disease (MONICA) classification. Therefore, with the approval of the ethical committee of Fukushima Medical University, we cross-referenced the death certificate data with data from the Fukushima Prefecture AMI registration survey, in which the diagnosis of AMI was based on the MONICA classification. ${ }^{12)}$ We defined all death certificate MI cases for which the patient's name, date of birth, and address accorded with the data from the AMI registration survey as MI cases. Other death certificate MI cases were defined as MI suspected (MIS) cases. Deaths from cancer or senility were excluded from the study. For each area, we tallied the number of people who fell into the SD, MI, and MIS groups.

Statistical analysis: We calculated the age-adjusted death rate for total, SD, and MI cases for indicative purposes, using the modeled population for 1985 for comparison, to compare the situations of death between areas. We obtained the population data from the monthly report for the
Table II. Age-Adjusted Incident Rates per 100,000 Individuals for Total Deaths and SDs in Each Area from 2011 to 2013

\begin{tabular}{|c|c|c|c|}
\hline & 2011 & 2012 & 2013 \\
\hline \multicolumn{4}{|l|}{ Total deaths } \\
\hline Area 1-3 & $73.4(1,584)^{\dagger}$ & $64.6(1,409)$ & $66.5(1,513)$ \\
\hline Area 1 & $69.7(181)$ & $45.6(114)$ & $50.6(118)$ \\
\hline Area 2 & $78.1(357)$ & $62.2(293)$ & $62.4(292)$ \\
\hline Area 3 & $72.6(1,046)$ & $67.0(1,002)$ & $70.1(1,103)$ \\
\hline \multicolumn{4}{|l|}{ SDs } \\
\hline Area 1-3 & $51.6(994)$ & $40.5^{*}(797)$ & $42.8^{*}(849)$ \\
\hline Area 1 & $38.2(88)$ & $27.1(61)$ & $32.9(68)$ \\
\hline Area 2 & $51.7(213)$ & 36.1 (163) & $39.9(167)$ \\
\hline Area 3 & $53.6(693)$ & $43.7 *(573)$ & $45.2 *(614)$ \\
\hline \multicolumn{4}{|l|}{ MI and MIS } \\
\hline Area 1-3 & $20.4(552)$ & $21.9(578)$ & $21.6(570)$ \\
\hline Area 1 & $32.1(43)$ & $26.2(45)$ & $23.2(43)$ \\
\hline Area 2 & 29.8 (128) & 33.7 (117) & 31.4 (107) \\
\hline Area 3 & $29.2(381)$ & $31.1(416)$ & $28.8(420)$ \\
\hline
\end{tabular}

$* P<0.05$ versus 2011. 'Population. MI indicates myocardial infarction; MIS, myocardial infarction suspected; and SD, sudden death.

study area, which is based on the Basic Resident Register for Fukushima Prefecture.

We used a Poisson regression model to evaluate the differences in the death rate for the total, SD, and MI groups across three years: 2011 (the base year), 2012, and 2013. Differences in the age-adjusted monthly death rates for each area were also evaluated using the Poisson regression model. The ages in this analysis were taken as the subject's age on January 1 of each year.

These analyses were conducted using SAS software version 9.4 (SAS Institute, Cary, NC, USA). $P$-values were two-sided, and $P$-values of $<0.05$ were considered to be statistically significant.

\section{Results}

We analyzed the death certificates of 26,177 people from 2013, 23,464 people from 2014, and 23,547 people from 2015. Because the number of MI cases was very small, we combined the MI and MIS groups (Table I).

In all areas, there were no significant differences in the age-adjusted death rate for the total group between 2011 and the following two years. Additionally, the results of the MI and MIS groups were the same as those of the total group, and there were no significant differences in the age-adjusted death rate for the MI and MIS groups between the years. However, the age-adjusted death rate of the SD group was significantly higher in 2011 in Area 1-3 than in 2012 or $2013(P<0.05$; Table II).

With respect to the comparison of the total death, $\mathrm{SD}$, and MI groups for each month and each area from January 2011 to December 2013, the total death rate was higher in Area 1 in March 2011, just after the disaster, than in the other two areas (Figure 2). However, this pattern was not seen in 2012 or 2013. The rate of SD was also higher in Area 1 than in the other areas in March 2011 (13.8 in Area 1, 9.1 in Area 2, and 7.6 in Area 3; P $<0.05$ ) (Figure 3). There were no significant differences in the age-adjusted death rates for the SD and MI groups in 2012 or 2013. 


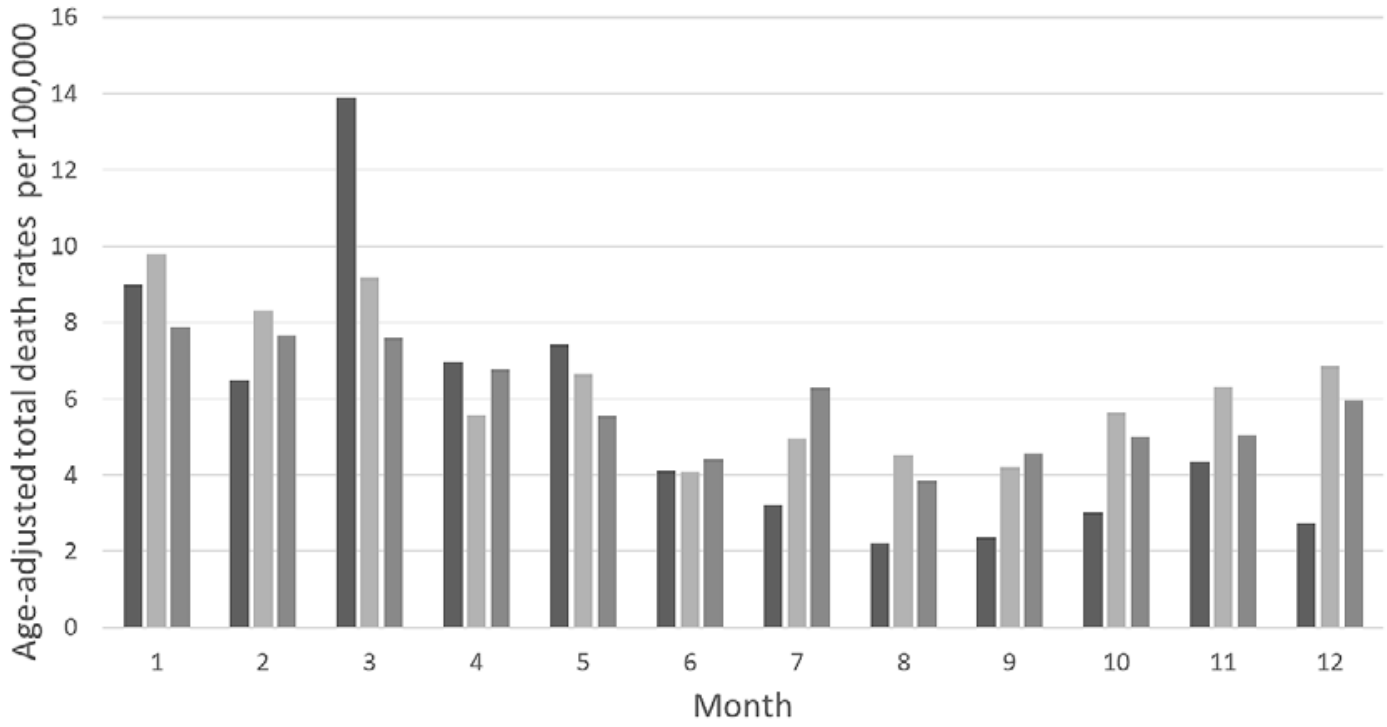

Area 1 Area 2 Area 3

Figure 2. Age-adjusted rates of the total death rate, which we extracted for each month, each area, and the period from January 2011 to December 2011.

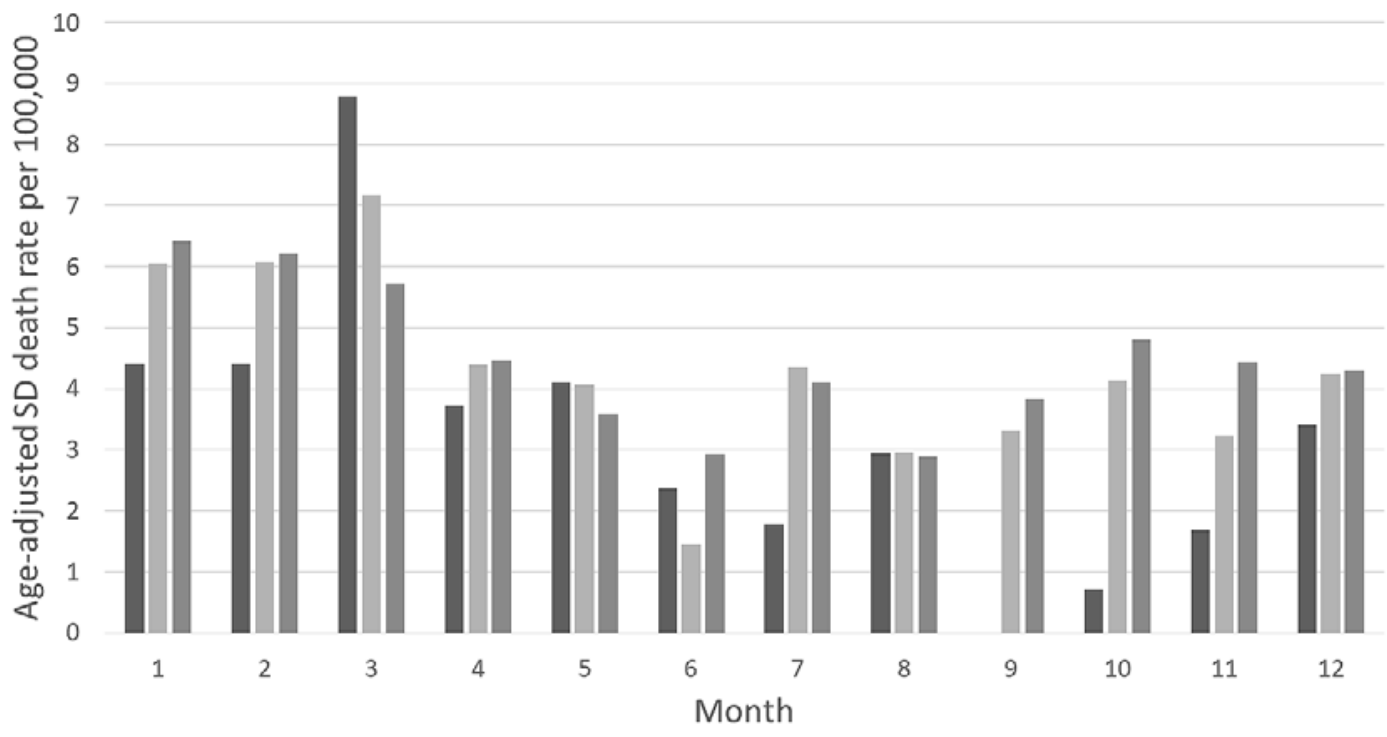

Area $1 \square$ Area $2 \square$ Area 3

Figure 3. Age-adjusted death rates of SDs for each month and each area, from January 2011 to December 2011.

\section{Discussion}

The incidence of cardiovascular diseases can increase at times of large-scale natural disasters as a result of mental and physical stress, and it has been reported that the incidence of AMI and SD increases following large-scale earthquakes. ${ }^{4-713)}$ In this study, we found that the incidence of SD increased in March 2011, just after the Great East Japan Earthquake. Further, the increase in March 2011 was greater in the population that was forced to evacuate following the nuclear power plant accident. Initially, we compared the population regulation of 2013 with that of 2011 in each area, and we found that the value was 91\% in Area 1, 92\% in Area 2, and $96 \%$ in Area 3. Thus, we believe that the movement of refugees had almost no influence on the population. Subsequently, we compared February with March 2011 in each area, and the ageadjusted death rate of SD showed a 99\% increase in Area $1,18 \%$ increase in Area 2, and $8 \%$ decrease in Area 3. Unfortunately, the data for 2010 were not available because we obtained the death certificates from the Fukushima Health Management Survey, which com- 
menced in 2011. Therefore, we compared February and January 2011 in each area (data before the earthquake). The rate of SD was higher in Area 3 than in Area 1. In the comparison of SD for each year and each area from 2011 to 2013, the rate of SD was higher in Area 3 than in Area 1; however, the reason was unclear, but it is thought that the incidence of SD was higher in Area 3 than in Area 1 before the disaster. We, therefore, think that the rate of SD tended to be higher in Area 1 than in the other areas in March 2011.

We did not, however, observe an increase in MI three years after the disaster. There was also no significant difference in the incidence of MI between the areas, although it may have been the cause of death in some of the SD cases.

Many people lost family members and their homes as a result of the earthquake and were then also forced to evacuate. In the evacuation area, there were inadequate daily supplies, such as water, electricity, and medicine. In addition, aftershocks with magnitude of over 5.0 were experienced for four weeks after the original earthquake. ${ }^{14)}$ In Fukushima, in particular, because of the power plant accident, the evacuation zone had to be enlarged, and the local residents lived in fear of radiation. In this situation, people, particularly those who lived in the evacuation zone, felt fear, uneasiness, depression, and physical and mental stress. Indeed, according to a mail survey, the nuclear accident caused psychological distress in the residents of Fukushima Prefecture. ${ }^{15)}$ Kunii, et al. reported that psychological distress in each evacuation zone was significantly positively associated with the radiation levels and that the power plant accident seriously affected the mental health of the residents. ${ }^{16)}$

The reason for the increase in SD and cardiovasculardisease-related death rate at the time of the disaster is thought to be related to the fact that stress increases the vasomotor reflex response and stimulation of the sympathetic nervous system. ${ }^{17,18)}$ The activation of the sympathetic nervous system causes an increase in blood pressure and heart rate. Indeed, it was reported that systolic and diastolic blood pressure increased subsequent to the Hanshin-Awaji earthquake. ${ }^{19)}$ Furthermore, Sato, et al. reported that, in Miyagi Prefecture, home blood pressure was significantly elevated immediately after the Great East Japan Earthquake. ${ }^{20)}$ In addition, the food provided in an evacuation shelter tends to be high in salt, to facilitate long-term preservation. Excessive salt intake causes high blood pressure. An increase in blood pressure and heart rate may result in a plaque rupture, which is associated with acute coronary syndrome. ${ }^{21,22)}$ Indeed, MI reportedly developed rapidly in a case with unstable plaque, following the 1994 Northridge earthquake.") Furthermore, arrhythmia can be caused by sudden environmental changes and heart vascular disorders, and aggravation and complications of the primary disease might be caused by medication interruptions. Because there were few toilets in the evacuation shelter, people reduced their water intake; consequently, they were more likely to experience dehydration. The acceleration of blood coagulation and the activation of platelets as a result of dehydration increase the risk of thrombosis and thrombotic disease. ${ }^{23)}$
Hence, this study suggests that the risk of stress for people at the evacuation zone was higher than for those at other disaster-stricken areas and that the resultant serious physical and mental stress caused SD, and possibly the onset of MI.

We examined the patterns of death and disease over the three years following the disaster, to investigate the long-term effects of the disaster. While there was an increase in SD immediately following the earthquake, this pattern did not continue over a longer timeframe. The Fukushima Health Management Survey suggests that there was an increase in lifestyle-related diseases, such as diabetes and dyslipidemia, and these are risk factors for the onset of cardiovascular disease. ${ }^{11,24)}$ Thus, it is necessary for us to continue investigating the onset of cardiovascular disease, as well as death from this disease, in the longterm. In addition, we should aim to reduce stress and the incidence of lifestyle-related diseases.

Strengths and limitations: Six years have passed since the Great East Japan Earthquake. Although the immediate effects of the earthquake have been well studied, there are no studies examining the effects of the disaster over a period of three years. In addition, to the best of our knowledge, this is the first study conducted that includes a comparison between the populations of the different areas forced to evacuate following this nuclear power plant accident.

Our study was based on the examination of death certificates. Although the authorities did their best to conduct thorough autopsies during the confusing situation following the disaster, it would have been difficult to accurately identify the cause of death in all cases. For example, some MI cases may have been recorded as "unknown cause of death" immediately following the earthquake.

In addition, the change in a person's registration address or permanent address before the disaster might not have always been noted. However, the refuge by the Fukushima Daiichi Nuclear Power Plant has issues regarding the compensation, and it is speculated that almost no change occurred, but we cannot deny the possibility that some people in Area 1 or Area 2 moved to Area 3. Therefore, the incidence of cardiovascular diseases in Area 1 and/or Area 2 may have been underestimated in this study.

Additionally, the rate of SD in 2012 and 2013 in Area 1 might have been estimated to be low because, in recent years, many solitary deaths have been occurring at evacuation shelters; and in this study, these might not have been counted as SD owing to late discovery at more than 24 hours.

Despite these limitations, in this study, we examined the long-term effects of the disaster, and the results can be used to suggest interventions that may improve the situation. As such, this study is important. In addition, our results will provide important information for people dealing with disasters in the future.

\section{Conclusions}

In this study, we examined the differences in the incidence of cardiovascular diseases between different areas of Fukushima Prefecture after the Great East Japan Earth- 
quake. As a result of the nuclear power plant accident, many people have been unable to return home and, therefore, remained as evacuees. Further follow-up surveys are, thus, needed in Fukushima Prefecture. This study suggests that the risk of stress for people at evacuation zones is higher than for those at other disaster-stricken areas and that the resultant serious physical and mental stress caused $\mathrm{SD}$, and possibly the onset of MI.

\section{Acknowledgments}

The authors would like to thank the staff of the Fukushima Health Management Survey for their important contributions. The findings and conclusions of this article are solely the responsibility of the authors and do not represent the official views of the Fukushima Prefecture government.

\section{Disclosure}

Conflicts of interest: None declared.

\section{References}

1. Japan Meteorological Agency. Monthly report on earthquake and volcanoes in Japan; 2011. Available at: http://www.data.jma.go.j p. Accessed August 16, 2019.

2. Fire and Disaster Management Agency. In: Tohoku District Pacific Offing Earthquake (the 155th Report). 2011.

3. Week Report of the Damage Situation of the East Japan Great Earthquake Disaster (the 1691st Report). Fukushima Revitalization Station.

4. Trichopoulos D, Katsouyanni K, Zavitsanos X, Tzonou A, Dalla-Vorgia P. Psychological stress and fatal heart attack: the Athens (1981) earthquake natural experiment. Lancet 1983; 1: 441-4.

5. Kloner RA, Leor J, Poole WK, Perritt R. Population-based analysis of the effect of the Northridge Earthquake on cardiac death in Los Angeles County, California. J Am Coll Cardiol 1997; 30: 1174-80

6. Suzuki S, Sakamoto S, Koide M, et al. Hanshin-Awaji earthquake as a trigger for acute myocardial infarction. Am Heart $\mathrm{J}$ 1997; 134: 974-7.

7. Aoki T, Fukumoto Y, Yasuda S, et al. The Great East Japan Earthquake Disaster and cardiovascular diseases. Eur Heart J 2012; 33: 2796-803.

8. Kitamura T, Kiyohara K, Iwami T. The great east Japan earthquake and out-of-hospital cardiac arrest. N Engl J Med 2013; 369: 2165-7.

9. Sado J, Kiyohara K, Iwami T, et al. Three-year follow-up after the great east Japan earthquake in the incidence of out-ofhospital cardiac arrest with cardiac origin. Circ J 2018; 82: 91922.

10. Vital Statistics Special Report of 2015. Ministry of Health, Labour and Welfare; 2017.

11. Ohira T, Nakano H, Nagai M, et al. Changes in cardiovascular risk factors after the great east Japan earthquake. Asia Pac J Public Health 2017; 29: 47-55S.

12. Nakazato K, Yamaki T, Kijima M, et al. Start and initial results of the Fukushima Prefecture acute myocardial infarction registration survey. Fukushima J Med Sci 2013; 59: 27-34.

13. Leor J, Poole WK, Kloner RA. Sudden cardiac death triggered by an earthquake. N Engl J Med 1996; 334: 413-9.

14. Yamaki T, Nakazato K, Kijima M, Maruyama Y, Takeishi Y. Impact of the Great East Japan Earthquake on acute myocardial infarction in Fukushima Prefecture. Disaster Med Public Health Prep 2014; 8: 212-9.

15. Yabe H, Suzuki Y, Mashiko H, et al. Psychological distress after the Great East Japan Earthquake and Fukushima Daiichi Nuclear Power Plant accident: results of a mental health and lifestyle survey through the Fukushima Health Management Survey in FY2011 and FY2012. Fukushima J Med Sci 2014; 60: 5767.

16. Kunii Y, Suzuki Y, Shiga T, et al. Severe psychological distress of evacuees in evacuation zone caused by the Fukushima Daiichi nuclear Power plant accident: the Fukushima health management survey. PLOS ONE 2016; 11: e0158821.

17. Huang JL, Chiou CW, Ting CT, Chen YT, Chen SA. Sudden changes in heart rate variability during the 1999 Taiwan earthquake. Am J Cardiol 2001; 87: 245-8, A9.

18. Tucker P, Pfefferbaum B, Jeon-Slaughter H, Khan Q, Garton T. Emotional stress and heart rate variability measures associated with cardiovascular risk in relocated Katrina survivors. Psychosom Med 2012; 74: 160-8.

19. Saito K, Kim JI, Maekawa K, Ikeda Y, Yokoyama M. The great Hanshin-Awaji earthquake aggravates blood pressure control in treated hypertensive patients. Am J Hypertens 1997; 10: 217-21.

20. Satoh M, Kikuya M, Ohkubo T, Imai Y. Acute and subacute effects of the great East Japan earthquake on home blood pressure values. Hypertension 2011; 58: e193-4.

21. Kario K, Matsuo T, Shimada K, Pickering TG. Factors associated with the occurrence and magnitude of earthquake-induced increases in blood pressure. Am J Med 2001; 111: 379-84.

22. Muller JE, Abela GS, Nesto RW, Tofler GH. Triggers, acute risk factors and vulnerable plaques: the lexicon of a new frontier. $\mathrm{J}$ Am Coll Cardiol 1994; 23: 809-13.

23. Kario K, Matsuo T, Kobayashi H, Yamamoto K, Shimada K. Earthquake-induced potentiation of acute risk factors in hypertensive elderly patients: possible triggering of cardiovascular events after a major earthquake. J Am Coll Cardiol 1997; 29: 926-33.

24. Suzuki H, Ohira T, Takeishi Y, et al. Increased prevalence of atrial fibrillation after the Great East Japan Earthquake: results from the Fukushima Health Management Survey. Int J Cardiol 2015; 198: 102-5 